\title{
Assertion-based Debugging of Higher-Order (C)LP Programs*
}

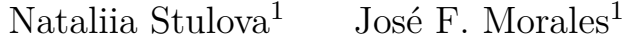 \\ ${ }^{1}$ IMDEA Software Institute \\ \{nataliia.stulova,josef.morales, manuel.hermenegildo\}@imdea.org
}

\author{
Manuel V. Hermenegildo ${ }^{1,2}$ \\ ${ }^{2}$ School of Computer Science \\ Technical University of Madrid (UPM) \\ manuel.hermenegildo@upm.es
}

\begin{abstract}
Higher-order constructs extend the expressiveness of firstorder (Constraint) Logic Programming ((C)LP) both syntactically and semantically. At the same time assertions have been in use for some time in (C)LP systems helping programmers detect errors and validate programs. However, these assertion-based extensions to (C)LP have not been integrated well with higher-order to date. This paper contributes to filling this gap by extending the assertion-based approach to error detection and program validation to the higher-order context within (C)LP. We propose an extension of properties and assertions as used in (C)LP in order to be able to fully describe arguments that are predicates. The extension makes the full power of the assertion language available when describing higher-order arguments. We provide syntax and semantics for (higher-order) properties and assertions, as well as for programs which contain such assertions, including the notions of error and partial correctness. We also discuss several alternatives for performing run-time checking of such programs.
\end{abstract}

Categories and Subject Descriptors D.1.6 [Programming Techniques]: Logic Programming; D.2.4 [Software Engineering]: Software/Program Verification-assertion checkers, validation; D.3.3 [Programming Languages]: Language Constructs and Features-constraints; F.3.1 [Logics and Meanings of Programs]: Specifying and Verifying and Reasoning about Programs - assertions, pre- and post-conditions, specification techniques; F.3.2 [Logics and Meanings of Programs]: Semantics of Programming Languages - operational semantics.

Keywords Logic Programming, Constraint Logic Programming, Higher-order, Validation, Assertions

\footnotetext{
* Research supported in part by projects EU FP7 318337 ENTRA, Spanish MINECO TIN2012-39391 StrongSoft and TIN2008-05624 DOVES, and Comunidad de Madrid TIC/1465 PROMETIDOS-CM.
}

Permission to make digital or hard copies of all or part of this work for personal or classroom use is granted without fee provided that copies are not made or distributed for profit or commercial advantage and that copies bear this notice and the full citation on the first page. Copyrights for components of this work owned by others than ACM must be honored. Abstracting with credit is permitted. To copy otherwise, or republish, to post on servers or to redistribute to lists, requires prior specific permission and/or a fee. Request permissions from permissions@acm.org.

PPDP '14, September 08-10, 2014, Canterbury, UK. Copyright @ 2014 ACM 978-1-nnnn-nnnn-n/yy/mm. . \$15.00 http://dx.doi.org/10.1145/nnnnnnn.nnnnnnn

\section{Introduction}

Higher-order programming adds flexibility to the software development process. Within the (Constraint) Logic Programming ((C)LP) paradigm, Prolog has included higherorder constructs since the early days, and there have many other proposals for combining the first-order kernel of (C)LP with different higher-order constructs (see, e.g., [5, 6, 8, 22, 23, 30.). Many of these proposals are currently in use in different (C)LP systems and have been found very useful in programming practice, inheriting the well-known benefits of code reuse (templates), elegance, clarity, and modularization.

A number of extensions have also been proposed for (C)LP in order to enhance the process of error detection and program validation. In addition to the use of classical strong typing [15, 27, a number of other approaches have been proposed which are based on the dynamic and/or static checking of user-provided, optional assertions [3, 4, 10, 13, 16, 21, 24, 26. In practice, different aspects of the model of [13, 26. have been incorporated in a number of widely-used (C)LP systems, such as Ciao, SWI, and XSB [14, 20, 28. A similar evolution is represented by the soft/gradual typing-based approaches in functional programming and the contractsbased extensions in object-oriented programming [7, 1719, 29.

These two aspects, assertions and higher-order, are not independent. When higher-order constructs are introduced in the language it becomes necessary to describe properties of arguments of predicates that are themselves also predicates. While the combination of contracts and higherorder has received some attention in functional programming [9, 11], within (C)LP the combination of higher-order with the previously mentioned assertion-based approaches has received comparatively little attention to date. Current Prolog systems simply use basic atomic types (i.e., stating simply that the argument is a pred, callable, etc.) to describe predicate-bearing variables. The approach of [2] is oriented to meta programming. It allows describing metatypes but there is no notion of directionality (modes), and only a single pattern is allowed per predicate.

This paper contributes to filling the existing gap between higher-order and assertions in (C)LP. Our starting point is the Ciao assertion model [13, 26], since, as mentioned before, it has been adopted at least in part in a number of the most popular (C)LP systems. After some preliminaries and notation (Section 2) we start by extending the traditional notion of programs and derivations in order to deal with higher-order calls and recall and adapt the notions of first- 
order conditional literals, assertions, program correctness, and run-time checking to this type of derivations (Section 3). This part allows us to revisit the traditional model in this new, higher-order context, while introducing a different formalization than the original one of [26]. This formalization, which will be used throughout the paper, is more compact and gathers all assertion violations as opposed to just the first one, among other differences. We then define an extension of the properties used in assertions and of the assertions themselves to higher-order, and provide corresponding semantics and results (Section 4 ).

\section{Preliminaries and Notation}

We recall some concepts and notation from standard (C)LP theory. We denote by VS, FS, and PS the set of variable, function, and predicate symbols, respectively. Variables start with a capital letter. Each $p \in$ PS and $f \in$ FS is associated to a natural number called its arity, written $\operatorname{ar}(p)$ or $\operatorname{ar}(f)$. The set of terms TS is inductivelly defined as follows: VS $\subset$ TS, if $f \in \mathrm{FS}$ and $t_{1}, \ldots, t_{n} \in \mathrm{TS}$ then $f\left(t_{1}, \ldots, t_{n}\right) \in$ TS where $\operatorname{ar}(f)=n$. An atom has the form $p\left(t_{1}, \ldots, t_{n}\right)$ where $p \in P S, \operatorname{ar}(p)=n$, and $t_{1}, \ldots, t_{n} \in T S$. A constraint is essentially a conjunction of expressions built from predefined predicates (such as term equations or inequalities over the reals) whose arguments are constructed using predefined functions (such as real addition). A literal is either an atom or a constraint. A goal is a finite sequence of literals. A rule is of the form $H:-B$ where $H$, the head, is an atom and $B$, the body, is a possibly empty finite sequence of literals. A constraint logic program, or program, is a finite set of rules.

We use $\sigma$ to represent a variable renaming and $\sigma(X)$ to represent the result of applying the renaming $\sigma$ to some syntactic object $X$ (a term, atom, literal, goal, etc.). The definition of an atom $A$ in a program, $\operatorname{defn}(A)$, is the set of variable renamings of the program rules such that each renaming has $A$ as a head and has distinct new local variables. We assume that all rule heads are normalized, i.e., $H$ is of the form $p\left(X_{1}, \ldots, X_{n}\right)$ where the $X_{1}, \ldots, X_{n}$ are distinct free variables. This is not restrictive since programs can always be normalized, and it facilitates the presentation. However, for conciseness in the examples we sometimes use non-normalized programs. Let $\bar{\exists}_{L} \theta$ be the constraint $\theta$ restricted to the variables of the syntactic object $L$. We denote constraint entailment by $\models$, so that $\theta_{1} \models \theta_{2}$ denotes that $\theta_{1}$ entails $\theta_{2}$. In such case we say that $\theta_{2}$ is weaker than $\theta_{1}$.

For brevity, we will assume in the rest of the paper that we are dealing with a single program, so that all sets of rules, etc. refer to that implicit program and it is not necessary to refer to it explicitly in the notation.

\subsection{Operational Semantics}

The operational semantics of a program is given in terms of its "derivations", which are sequences of reductions between "states". A state $\langle G \mid \theta\rangle$ consists of a goal $G$ and a constraint store (or store for short) $\theta$. We use :: to denote concatenation of sequences and we assume for simplicity that the underlying constraint solver is complete. We use $S \rightsquigarrow S^{\prime}$ to indicate that a reduction can be applied to state $S$ to obtain state $S^{\prime}$. Also, $S \rightsquigarrow^{*} S^{\prime}$ indicates that there is a sequence of reduction steps from state $S$ to state $S^{\prime}$. We denote by $D_{[i]}$ the $i$-th state of the derivation. As a shorthand, given a non-empty derivation $D, D_{[-1]}$ denotes the last state. A query is a pair $(L, \theta)$, where $L$ is a literal and $\theta$ a store, for which the $(\mathrm{C}) \mathrm{LP}$ system starts a computation from state $\langle L \mid \theta\rangle$. The set of all derivations from the query $Q$ is denoted $\operatorname{derivs}(Q)$. The observational behavior of a program is given by its "answers" to queries. A finite derivation from a query $(L, \theta)$ is finished if the last state in the derivation cannot be reduced. Note that derivs $(Q)$ contains not only finished derivations but also all intermediate derivations from a query. A finished derivation from a query $(L, \theta)$ is successful if the last state is of the form $\left\langle\square \mid \theta^{\prime}\right\rangle$, where $\square$ denotes the empty goal sequence. In that case, the constraint $\bar{\exists}_{L} \theta^{\prime}$ is an answer to $S$. We denote by answers $(Q)$ the set of answers to a query $Q$. A finished derivation is failed if the last state is not of the form $\langle\square \mid \theta\rangle$. A query $Q$ finitely fails if derivs $(Q)$ is finite and contains no successful derivation.

\section{First-order Assertions on Higher-order Derivations}

\subsection{Higher-order Programs and Derivations}

We start by extending the definition of program, state reduction, and derivations in order to deal with the syntax and semantics of higher-order calls.

Definition 1. Higher-order programs are a generalization of constraint logic programs where:

- The set of literals $L S$ is extended to include higher-order literals $X\left(t_{1}, \ldots, t_{n}\right)$, where $X \in V S$ and the $t_{i} \in T S$.

- The set of terms TS is extended so that PS $\subset$ TS (i.e., predicate symbols $p$ can be used as constants).

In the following we assume a simple semantics where when a call to a higher-order literal $X\left(t_{1}, \ldots, t_{n}\right)$ occurs, $X$ has to be constrained to a predicate symbol in the store ${ }^{1}$

Definition 2. A state $S=\langle L:: G \mid \theta\rangle$ where $L$ is a literal can be reduced to a state $S^{\prime}$, denoted $S \rightsquigarrow S^{\prime}$, as follows:

1. If $L$ is a constraint and $\theta \wedge L$ is satisfiable, then $S^{\prime}=$ $\langle G \mid \theta \wedge L\rangle$.

2. If $L$ is an atom of the form $p\left(t_{1}, \ldots, t_{n}\right)$, for some rule $(L:-B) \in \operatorname{defn}(L)$, then $S^{\prime}=\langle B:: G \mid \theta\rangle$.

3. If $L$ is of the form $X\left(t_{1}, \ldots, t_{n}\right)$, then $S^{\prime}=\left\langle G^{\prime} \mid \theta\right\rangle$ where either $G^{\prime}=p\left(t_{1}, \ldots, t_{n}\right):: G$ if $\exists p \in P S \wedge \theta$ $(X=p) \wedge \operatorname{ar}(p)=n$, or $G^{\prime}=\epsilon_{\text {uninst_call }}$ otherwise.

The concepts of answers and of finished and successful derivations carry over without change to this notion of higher-order derivations. The notion of (finitely) failed derivation is extended as follows:

Definition 3. A finished derivation from a query $(L, \theta)$ is failed iff its last state is not of the form $\left\langle\square \mid \theta^{\prime}\right\rangle$ or $\left\langle\epsilon_{\text {uninst_call }} \mid \theta\right\rangle$.

Finally, we introduce the concept of floundered derivations:

Definition 4. A finished derivation from a query $(L, \theta)$ is floundered iff its last state is of the form $\left\langle\epsilon_{\text {uninst_call }} \mid \theta\right\rangle$.

\subsection{First-order Pred Assertions}

Assertions are linguistic constructions for expressing properties of programs. They are used for detecting deviations of

\footnotetext{
${ }^{1}$ This is also the most frequent semantics in current systems. Other alternatives, such as residuation [1] (delays), predicate enumeration, etc. can also be used, requiring relatively straightforward adaptations of the model proposed.
} 
the program behavior (symptoms) with respect to such assertions, or to ensure that no such deviations exist (correctness). Herein, we will use the pred assertions of 25], given that they are the most frequently used assertions in practice, and they subsume the other assertion schemas in that language. Thus, in the following we will use simply the term assertion to refer to a pred assertion. Assertions allow specifying certain conditions on the constraint store that must hold at certain points of program derivations. In particular, they allow stating sets of preconditions and conditional postconditions for a given predicate. A set of assertions for a predicate is of the form:

$$
\begin{aligned}
& \text { :- pred Head : } \text { Pre }_{1} \Rightarrow \text { Post }_{1} . \\
& \text { ․ } \\
& \text { :- pred Head : Pre } \text { Pre }_{n} \Rightarrow \text { Post }_{n} .
\end{aligned}
$$

where Head is a normalized atom that denotes the predicate that the assertions apply to, and the Pre $_{i}$ and Post ${ }_{i}$ refer to the variables of Head. We assume that variables in assertions are renamed such that the Head atom is identical for all assertions for a given predicate. A set of assertions as above states that in any execution state $\langle$ Head :: $G \mid \theta\rangle$ at least one of the Pre $_{i}$ conditions should hold, and that, given the $\left(\right.$ Pre $_{i}$, Post $\left._{i}\right)$ pair(s) where Pre $_{i}$ holds, then, if

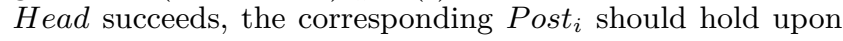
success. The following example illustrates the basic concepts involved:

Example 1. The procedure qsort $(\mathrm{A}, \mathrm{B})$ is the usual one that relates lists $\mathrm{A}$ and their sorted versions $\mathrm{B}$. The following assertions:

:- pred qsort(A,B) : list(A) $\Rightarrow$ sorted(B), list(B).

$:-\operatorname{pred} q \operatorname{sort}(A, B): \operatorname{list}(B) \Rightarrow$ permutation $(B, A), \operatorname{list}(A)$.

state that (restrict the meaning of qsort to):

- qsort(A,B) should be called either with A constrained to a list or with B constrained to a list;

- if qsort(A,B) succeeds when called with A constrained to a list then on success $\mathrm{B}$ should be a sorted list; and

- if qsort(A,B) succeeds when called with B constrained to a list then on success A should be a list which is a permutation of $\mathrm{B}$.

\subsection{Conditions on the Constraint Store}

The conditions on the constraint store used in assertions are specified by means of special literals (e.g., list(A), sorted (B), list (B), and permutation (B, A) in the previous example) that we will herein call prop literals. More concretely, we assume the Pre ${ }_{i}$ and Post ${ }_{i}$ to be DNF formulas of such literals.

We also assume that for each prop literal $L_{p}$ used in some assertion there exists a corresponding predicate $p$ defining it. Then, we can define the meaning of prop literals as follows:

Definition 5. The meaning of a prop literal $L_{p}$ defined by predicate $p$, denoted $\left|L_{p}\right|$, is the set of constraints given by answers $\left(\left(L_{p}\right.\right.$, true $\left.)\right)$.

Intuitively, the meaning of prop literals is the set of "weakest" constraints for which the literal holds:

Example 2. Prop literals list/1 and sorted/1 can be defined by:

list ([]).

$\left.\operatorname{sorted}\left([]_{-}\right]\right)$.

$$
\text { list ([_|L]) :- list (L). } \quad \operatorname{sorted}([]) \text {. }
$$$$
\operatorname{sorted}([X, Y \mid L]):-X=<Y, \operatorname{sorted}([Y \mid L]) \text {. }
$$

Then, their meaning is given by $|\operatorname{list}(A)|=\{A=[], A=$ $[B \mid C] \wedge \operatorname{list}(C)\}$ and $|\operatorname{sorted}(A)|=\{A=[], A=[B], A=$ $[B, C \mid D] \wedge B \leq C \wedge E=[C \mid D] \wedge \operatorname{sorted}(E)\}$.
The following definition from 26] defines when the condition represented by a prop literal (defined by a program predicate) holds for a given store:

Definition 6. A prop literal $L$ succeeds trivially for $\theta$, denoted $\theta \Rightarrow_{P} L$, iff $\exists \theta^{\prime} \in$ answers $((L, \theta))$ such that $\theta=\theta^{\prime}$. A DNF formula of prop literals succeeds trivially for $\theta$ if all of the prop literals of at least one conjunct of the formula succeeds trivially.

Intuitively, a prop literal $L$ succeeds trivially if $L$ succeeds for $\theta$ without adding new "relevant" constraints to $\theta$ :

Example 3. Consider prop literals list $(A)$ and $\operatorname{sorted}(B)$ and the predicate definitions of Example 2

- Assume that $\theta=(A=f)$. Since $\forall \theta^{\prime} \in|\operatorname{list}(A)|: \theta \not \models \theta^{\prime}$, as we would expect, $\theta \nRightarrow_{P} \operatorname{list}(A)$.

- Assume now that $\theta=\left(A=\left[\_\mid X s\right]\right)$. Though $A$ is compatible with a list, it is not actually a (nil terminated) list. Again in this case $\forall \theta^{\prime} \in|\operatorname{list}(A)|: \theta \not \forall \theta^{\prime}$ and thus again $\theta \nRightarrow_{P}$ list $(A)$. The intuition behind this is that we cannot guarantee that $A$ is actually a list given $\theta$, since a possible instance of $A$ in $\theta$ is $A=[\ldots \mid f]$, which is clearly not a list.

- Finally, assume that $\theta=(A=[B] \wedge B=1)$. In such case $\exists \theta^{\prime}=(A=[B \mid C] \wedge C=[])$ such that $\theta=\theta^{\prime}$ and $\exists c=(B=1)$ such that $\left(c \wedge \theta^{\prime} \not \forall\right.$ false $) \wedge\left(\theta^{\prime} \wedge c \mid=\theta\right)$. Thus, in this last case $\theta \Rightarrow_{P} \operatorname{list}(A)$.

This means that we are considering prop literals as instantiation checks [12, 25]: they are true iff the variables they check for are at least as constrained as their predicate definition requires.

Definition 7. A prop literal $L$ is a test iff $\forall \theta$ either $\theta \Rightarrow_{P} L$ or $(L, \theta)$ finitely fails.

\subsection{First-order Assertion Conditions and their Semantics}

We represent the different checks on the constraint store imposed by a set of assertions as a set of assertion conditions as follows.

Definition 8. Given a predicate represented by a normalized atom Head, if the corresponding set of assertions is $\mathcal{A}=$ $\left\{A_{1} \ldots A_{n}\right\}$, with $A_{i}=":-$ pred Head : Pre ${ }_{i} \Rightarrow$ Post $_{i} . "$ the set of assertion conditions for Head is $\left\{C_{0}, C_{1}, \ldots, C_{n}\right\}$, with:

$$
C_{i}= \begin{cases}\text { calls }\left(\text { Head }, \bigvee_{j=1}^{n} \text { Pre }_{j}\right) & i=0 \\ \text { success }\left(\text { Head }, \text { Pre }_{i}, \text { Post }_{i}\right) & i=1 . . n\end{cases}
$$

If there are no assertions associated with Head then the corresponding set of conditions is empty. The set of assertion conditions for a program is the union of the assertion conditions for each of the predicates in the program. Also, given a single assertion $A_{i}$ we define its corresponding set of assertion conditions as $\left\{C_{0}, C_{i}\right\}$ (this will be useful in defining the status of an assertion).

The calls $(H e a d, \ldots)$ conditions encode the checks that the calls to the predicate represented by Head are within those admissible by the set of assertions, and we thus call them the calls assertion conditions. The success $\left(\right.$ Head $_{i}$, Pre $_{i}$, Post $\left._{i}\right)$ conditions encode the checks for compliance of the successes for particular sets of calls, and we thus call them the success assertion conditions. 
Example 4. The assertion conditions corresponding to the predicate assertions for qsort in Example 1 are as follows:

calls $(q \operatorname{sort}(A, B),(\operatorname{list}(A), \operatorname{list}(B)))$

$\operatorname{success}(q \operatorname{sort}(A, B), \operatorname{list}(A),(\operatorname{sorted}(B), \operatorname{list}(B)))$

success $(q \operatorname{sort}(A, B), \operatorname{list}(B),($ permutation $(B, A), \operatorname{list}(A)))$

In order to define the semantics of assertion conditions, we introduce the auxiliary partial functions prestep and step as follows:

$$
\begin{aligned}
\operatorname{prestep}\left(L_{a}, D\right)=(\theta, \sigma) \equiv & D_{[-1]}=\langle L:: G \mid \theta\rangle \wedge \exists \sigma L=\sigma\left(L_{a}\right) \\
\operatorname{step}\left(L_{a}, D\right)=\left(\theta, \sigma, \theta^{\prime}\right) \equiv & D_{[-1]}=\left\langle G \mid \theta^{\prime}\right\rangle \wedge \exists \sigma L=\sigma\left(L_{a}\right) \\
& \wedge \exists i D_{[i]}=\langle L:: G \mid \theta\rangle
\end{aligned}
$$

Given a derivation whose current state is a call to $L_{a}$ (normalized atom), the prestep function returns the substitution $\sigma$ for $L_{a}$, and the constraint store $\theta$ at the predicate call (i.e., just before the literal is reduced). Given a derivation whose current state corresponds exactly to the return from a call to $L_{a}$, the step function returns the substitution $\sigma$ for $L_{a}$, the constraint store $\theta$ at the call to $L_{a}$, and the constraint store $\theta^{\prime}$ at $L_{a}$ 's success (i.e., just after all literals introduced from the body of $L_{a}$ have been fully reduced). Using these functions, the semantics of our calls and success assertion conditions are given by the following definition:

Definition 9. Given a calls or success assertion condition $C$, the valuation of $C$ on a derivation $D$, denoted solve $(C, D)$ is defined as follows:

$$
\begin{aligned}
\text { solve }\left(\text { calls }\left(L_{a}, \text { Pre }\right), D\right) \equiv & \left(\operatorname{prestep}\left(L_{a}, D\right)=(\theta, \sigma)\right) \\
& \Rightarrow\left(\theta \Rightarrow_{P} \sigma(\text { Pre })\right) \\
\operatorname{solve}\left(\operatorname{success}\left(L_{a}, \text { Pre }, \text { Post }\right), D\right) \equiv & \left(\operatorname{step}\left(L_{a}, D\right)=\left(\theta, \sigma, \theta^{\prime}\right)\right) \\
& \Rightarrow\left(\left(\theta \Rightarrow_{P} \sigma(\text { Pre })\right)\right. \\
& \left.\Rightarrow\left(\theta^{\prime} \Rightarrow_{P} \sigma(\text { Post })\right)\right)
\end{aligned}
$$

where $L_{a}$ is a normalized atom.

\subsection{Status of Assertions and Partial Correctness}

As mentioned before, the intended use of our assertions is to perform debugging with respect to partial correctness, i.e., to ensure that the program does not produce unexpected results for valid ("expected") queries ${ }^{2}$ Thus, we extend our notion of program to include assertions and valid queries.

Definition 10. An annotated program is a tuple $(P, \mathcal{Q}, \mathcal{A})$ where $P$ is a (higher-order) constraint logic program (as defined in Section 2), $\mathcal{Q}$ is a set of valid queries, and $\mathcal{A}$ is a set of assertions. As before, $\mathcal{A}_{C}$ denotes the set of calls and success assertion conditions derived from $\mathcal{A}$.

In the context of annotated programs we extend derivations to operate on the set of valid queries as follows: $\operatorname{derivs}(\mathcal{Q})=$ $\bigcup_{Q \in \mathcal{Q}} \operatorname{derivs}(Q)$. We now provide several simple definitions which will be instrumental:

Definition 11. Given the set of queries $\mathcal{Q}$, the assertion condition $C$ can be either checked or false, as follows:

$$
\begin{aligned}
\operatorname{checked}(C) & \equiv \forall D \in \operatorname{derivs}(\mathcal{Q}) . \operatorname{solve}(C, D) \\
\text { false }(C) & \equiv \exists D \in \operatorname{derivs}(\mathcal{Q}) \mid \neg \operatorname{solve}(C, D)
\end{aligned}
$$

\footnotetext{
${ }^{2}$ In practice, this set of expected queries is determined from module interfaces that define the set of exported predicates.
}

Definition 12. In an annotated program $(P, \mathcal{Q}, \mathcal{A})$ an assertion $A \in \mathcal{A}$ is checked (false) if all (any) of the corresponding assertion conditions are checked (false).

Definition 13. An annotated program $(P, \mathcal{Q}, \mathcal{A})$ is partially correct w.r.t. the set of assertions $\mathcal{A}$ and the set of queries $\mathcal{Q}$ iff $\forall A \in \mathcal{A}, A$ is checked for $\mathcal{Q}$.

Note that it follows immediately that a program is partially correct if all its assertion conditions are checked. The goal of assertion checking is thus to determine whether each assertion $A$ is false or checked for $\mathcal{Q}$. Again, for this it is sufficient to prove the corresponding assertions conditions false or checked. There are two kinds of approaches to doing this (which can also be combined). While it is in general not possible to try all derivations stemming from $\mathcal{Q}$, an alternative is to explore a hopefully representative set of them 21. Though this does not allow fully validating the program in general, it makes it possible to detect many incorrectness problems. This approach is explored in Section 3.6 in the context of our higher-order derivations. The second approach is to use global analysis techniques and is based on computing safe approximations of the program behavior statically 4, 13. The extension of this approach to higher-order assertions is beyond the scope of this paper.

\subsection{Operational Semantics for Higher-order Programs with First-order Assertions}

We now provide an operational semantics which checks whether assertion conditions hold or not while computing the (possibly higher-order) derivations from a query.

Definition 14. Given the atom $L_{a}$ and the set of assertion conditions $\mathcal{A}_{C}, \mathcal{A}_{C}^{\#}\left(L_{a}\right)$ denotes the set of labeled assertion condition instances for $L_{a}$ of the form $c \# C_{a}$, such that $\exists C \in$ $\mathcal{A}_{C}, C=\operatorname{calls}(L$, Pre $)$ (or $C=\operatorname{success}(L$, Pre, Post $\left.)\right), \sigma$ is a renaming s.t. $L=\sigma\left(L_{a}\right), C_{a}=$ calls $\left(L_{a}, \sigma(\right.$ Pre $)$ ) (or $C_{a}=\operatorname{success}\left(L_{a}, \sigma(\right.$ Pre $), \sigma($ Post $\left.\left.)\right)\right)$, and $c$ is an identifier that is unique for each $C_{a}$.

In order to keep track of the violated assertion conditions, we introduce an extended program state of the form $\langle G|$ $\theta|\mathcal{E}\rangle$, where $\mathcal{E}$ denotes the set of identifiers for falsified assertion condition instances. We also extend the set of literals with syntactic objects of the form check $(c)$ where $c$ is an identifier for an assertion condition instance, which we call check literals. Thus, a literal is now a constraint, an atom, a higher-order literal, or a check literal $]^{3}$

Definition 15. A state $S=\langle L:: G|\theta| \mathcal{E}\rangle$, where $L$ is a literal can be reduced to a state $S^{\prime}$, denoted $S \rightsquigarrow \mathcal{A} S^{\prime}$, as follows:

1. If $L$ is a constraint or $L=X\left(t_{1}, \ldots, t_{n}\right)$, then $S^{\prime}=\left\langle G^{\prime}\right|$ $\theta^{\prime}|\mathcal{E}\rangle$ where $G^{\prime}$ and $\theta^{\prime}$ are obtained in a same manner as in $\langle L:: G \mid \theta\rangle \rightsquigarrow\left\langle G^{\prime} \mid \theta^{\prime}\right\rangle$

2. If $L$ is an atom and $\exists(L:-B) \in \operatorname{defn}(L)$, then $S^{\prime}=\langle B$ :: PostC :: $\left.G|\theta| \mathcal{E}^{\prime}\right\rangle$ where:

- PostC is the sequence $\operatorname{check}\left(c_{1}\right)$ :: . . :: check $\left(c_{n}\right)$ including all the checks check $\left(c_{i}\right)$ such that $c_{i} \# \operatorname{success}\left(L\right.$, Pre $_{i}$, Post $\left._{i}\right) \in \mathcal{A}_{C}^{\#}(L) \wedge \theta \Rightarrow_{P}$ Pre $_{i}$

\footnotetext{
${ }^{3}$ While check literals are simply instrumental here, note that they are also directly useful for supporting program point assertions (which are basically check literals that appear in the body of rules) 25. However, for simplicity we do not discuss program point assertions in this paper.
} 
- and either $\mathcal{E}^{\prime}=\mathcal{E} \cup\{\bar{c}\}$ if $\exists c \#$ calls $(L$, Pre $) \in \mathcal{A}_{C}^{\#}(L)$ s.t. $\theta \nRightarrow_{P}$ Pre or $\mathcal{E}^{\prime}=\mathcal{E}$ otherwise.

3. If $L$ is a check literal check $(c)$, then $S^{\prime}=\left\langle G|\theta| \mathcal{E}^{\prime}\right\rangle$ where either $\mathcal{E}^{\prime}=\mathcal{E} \cup\{\bar{c}\}$ if $c \# \operatorname{success}\left(L,{ }_{-}\right.$, Post $) \in$ $\mathcal{A}_{C}^{\#}(L) \wedge \theta \nRightarrow_{P}$ Post or $\mathcal{E}^{\prime}=\mathcal{E}$ otherwise.

Note that the order in which the PostC check literals are selected is irrelevant.

The set of derivations for a program from its set of queries $\mathcal{Q}$ using the semantics with assertions is denoted $\operatorname{derivs}_{\mathcal{A}}(\mathcal{Q})$.

Definition 16. The set of error-erased derivations from $\rightsquigarrow \mathcal{A}$ is obtained by a syntactic rewriting $(-)^{\circ}$ that removes states that begin by a check literal, check literals from goals, and the error set. It is recursively defined as follows:

$$
\begin{aligned}
\left\{D_{1}, \ldots, D_{n}\right\}^{\circ} & =\left\{D_{1}^{\circ}, \ldots, D_{n}^{\circ}\right\} \\
\left(S_{1}, \ldots, S_{m}, S_{m+1}\right)^{\circ} & =\left\{\begin{array}{c}
\left(S_{1}, \ldots, S_{m}\right)^{\circ} \\
\text { if } S_{m+1}=\left\langle\operatorname{check}(-)::-S_{-}|-|-\right\rangle \\
\left(S_{1}, \ldots, S_{m}\right)^{\circ} \|\left(\left(S_{m+1}\right)^{\circ}\right) \\
\text { otherwise }
\end{array}\right. \\
\langle G|\theta| \mathcal{E}\rangle^{\circ} & =\left\langle G^{\circ} \mid \theta\right\rangle \\
(L:: G)^{\circ} & = \begin{cases}G^{\circ} & \text { if } L=\text { check }(-) \\
L::\left(G^{\circ}\right) & \text { otherwise }\end{cases}
\end{aligned}
$$

where || stands for sequence concatenation.

Theorem 1 (Correctness and Completeness Under Assertion Checking). For any annotated program $(P, \mathcal{Q}, \mathcal{A})$, given $\mathcal{D}=\operatorname{derivs}(\mathcal{Q})$ and $\mathcal{D}^{\prime}=\operatorname{derivs}_{\mathcal{A}}(\mathcal{Q})$, it holds that $\mathcal{D}$ and $\mathcal{D}^{\prime}$ are equivalent after filtering out check literals and error sets (formally defined as $\mathcal{D}=\left(\mathcal{D}^{\prime}\right)^{\circ}$ in appendix Def. 16).

Proof. We will prove $\mathcal{D}=\left(\mathcal{D}^{\prime}\right)^{\circ}$ by showing that $\mathcal{D} \subseteq\left(\mathcal{D}^{\prime}\right)^{\circ}$ and $\mathcal{D} \supseteq\left(\mathcal{D}^{\prime}\right)^{\circ}$.

- () For all $D \in \mathcal{D}$ exists $D^{\prime} \in \mathcal{D}^{\prime}$ so that $D=\left(D^{\prime}\right)^{\circ}$.

- () For all $D^{\prime} \in \mathcal{D}^{\prime}, D=\left(D^{\prime}\right)^{\circ} \in \mathcal{D}$.

We will prove each case:

- (C) Let $D=\left(S_{1}, \ldots, S_{n}\right), S_{i}=\left\langle L_{i} \mid \theta_{i}\right\rangle$, for some $Q=\left(L_{1}, \theta_{1}\right) \in \mathcal{Q}$ and $S_{i} \rightsquigarrow S_{i+1}$. Proof by induction on the length $n$ of $D$ :

- Base case $(n=1)$. Let $S_{1}^{\prime}=\left\langle L_{1}\left|\theta_{1}\right| \emptyset\right\rangle$. It holds that $\left.\left(S_{1}^{\prime}\right)^{\circ}=\left\langle L_{1}\left|\theta_{1}\right| \emptyset\right\rangle\right)^{\circ}=\left\langle L_{1}^{\circ} \mid \theta_{1}\right\rangle=\left\langle L_{1} \mid \theta_{1}\right\rangle=S_{1}$ (since $L_{1}$ does not contain any check literal). Thus, $\left(D^{\prime}\right)^{\circ}=\left(\left(S_{1}^{\prime}\right)\right)^{\circ}=\left(\left(S_{1}^{\prime}\right)^{\circ}\right)=\left(S_{1}\right)=D$.

- Inductive case (show $n+1$ assuming $n$ holds). For each $D_{2}=\left(S_{1}, \ldots, S_{n}, S_{n+1}\right)$ there exists $D_{2}^{\prime}=$ $\left(S_{1}^{\prime}, \ldots, S_{m}^{\prime}, S_{m+1}^{\prime}\right)$ such that $\left(D_{2}^{\prime}\right)^{\circ}=D_{2}$. Given the induction hypothesis it is enough to show that for each $S_{n} \rightsquigarrow S_{n+1}$ there exists $S_{m}^{\prime} \rightsquigarrow \mathcal{A} S_{m+1}^{\prime}$, such that $\left(S_{m+1}^{\prime}\right)^{\circ}=S_{n+1}$. According to $\rightsquigarrow \mathcal{A}$ (see Def. 15, $L_{m+1}^{\prime}$ and $\theta_{m+1}^{\prime}$ are obtained in the same way than in $\rightsquigarrow$ (see Def. 2), except for the introduction of check literals. Since all check literals are removed in errorerased states, it follows that $\left(S_{m+1}^{\prime}\right)^{\circ}=S_{n+1}$.

- (つ) Let $D^{\prime}=\left(S_{1}^{\prime}, \ldots, S_{m}^{\prime}\right), S_{i}^{\prime}=\left\langle L_{i}^{\prime}\left|\theta_{i}^{\prime}\right| \mathcal{E}_{i}\right\rangle$, for some $Q=\left(L_{1}^{\prime}, \theta_{1}^{\prime}\right) \in \mathcal{Q}$ and $S_{i}^{\prime} \rightsquigarrow \mathcal{A} S_{i+1}^{\prime}$. Proof by induction on the length $m$ of $D^{\prime}$ :

- Base case $(m=1)$. It holds that $\left(S_{1}^{\prime}\right)^{\circ}=S_{1}$ (showed in base case for $\subseteq$ ). Then $\left(D^{\prime}\right)^{\circ}=D \in \mathcal{D}$.
- Inductive case (show $m+1$ assuming $m$ holds). We want to show that given $D_{2}^{\prime}=\left(S_{1}^{\prime}, \ldots, S_{m}^{\prime}, S_{m+1}^{\prime}\right)$, $\left(D_{2}^{\prime}\right)^{\circ}=D_{2} \in \mathcal{D}$. Given the induction hypothesis it is enough to show that for each $S_{m}^{\prime} \rightsquigarrow \mathcal{A} S_{m+1}^{\prime}$ there exists $S_{n} \rightsquigarrow S_{n+1}$ such that $S_{n+1}=\left(S_{m+1}^{\prime}\right)^{\circ}$ (so that $\left(S_{1}, \ldots, S_{n}, S_{n+1}\right) \in \mathcal{D}$ ) or $S_{n}=\left(S_{m+1}^{\prime}\right)^{\circ}$ $\left(D_{2}=D \in \mathcal{D}\right)$. According to cases of Def. 15

- If $L_{m}^{\prime}$ begins with a check literal then $\left(L_{m+1}^{\prime}\right)^{\circ}=$ $\left(L_{m}^{\prime}\right)^{\circ}$. Thus $\left(S_{m+1}^{\prime}\right)^{\circ}=\left(S_{m}^{\prime}\right)^{\circ}=S_{n}$.

- Otherwise, it holds that $\left(S_{m+1}^{\prime}\right)^{\circ}=S_{n+1}$ using the same reasoning than in the inductive case for $\subseteq$.

This result implies that the semantics with assertions can also be used to obtain all answers to the original query. Furthermore, the following theorem guarantees that we can use the proposed operational semantics for annotated programs in order to detect (all) violations of assertions:

Definition 17. Let $\mathcal{E}(D)$ denote the error set of the last state of derivation $D, D_{[-1]}=\left\langle\left._{-}\right|_{-} \mid \mathcal{E}\right\rangle$. The run-time valuation of an assertion condition $C$ on a derivation $D$ is given by:

$$
\begin{aligned}
\text { rtsolve }(C, D) \equiv & \forall c, C^{\prime}, \sigma, L\left(c \# C^{\prime} \in \mathcal{A}_{C}^{\#}(L) \wedge \sigma(C)=C^{\prime}\right) \\
& \Rightarrow \mathcal{E}(D) \nvdash \bar{c}
\end{aligned}
$$

I.e., condition rtsolve $(C, D)$ is valid if none of the possible instances of the assertion condition $C$ are in the error set for derivation $D$

Theorem 2 (Run-time Error Detection). For any annotated program $(P, \mathcal{Q}, \mathcal{A}), C \in \mathcal{A}_{C}$ is false iff $\exists D \in$ $\operatorname{derivs}_{\mathcal{A}}(\mathcal{Q})$ s.t. $\neg$ rtsolve $(C, D)$.

Proof. $A \in \mathcal{A}_{C}$ is false $\Leftrightarrow$ from Def. 12 and Def. $8 \exists\left\{C_{c}, C_{s}\right\}$ assertion conditions s.t. false $\left(C_{c}\right) \vee$ false $\left(C_{s}\right)$, where $C_{c}=\operatorname{calls}(L$, Pre $)$ and $C_{s}=$ success $(L$, Pre, Post) correspond to $A$. Let us first prove $\neg$ rtsolve $\left(C_{c}, D\right)$, and then $\neg$ rtsolve $\left(C_{s}, D\right)$.

false $\left(C_{c}\right)$

$\Leftrightarrow$ from Def. $11 \exists D \in \operatorname{derivs}(\mathcal{Q})$ s.t. $\neg \operatorname{solve}\left(C_{c}, D\right)$

$\Leftrightarrow$ from Def. 9 prestep $(L, D)=(\theta, \sigma) \wedge \theta \nRightarrow_{P} \sigma($ Pre $\left.)\right)$

$\Leftrightarrow$ from Def. $15 . \exists \rightsquigarrow \mathcal{A} S^{\prime}$ where:

$$
\begin{aligned}
S & =\langle L:: G|\theta| \mathcal{E}\rangle \text { s.t. } \exists c \# \text { calls }(L, \text { Pre }) \in \mathcal{A}_{C}^{\#}(L) \\
S^{\prime} & =\left\langle-|\theta| \mathcal{E}^{\prime}\right\rangle \wedge \mathcal{E}^{\prime}=\mathcal{E} \cup\{\bar{c}\}
\end{aligned}
$$

$\Leftrightarrow$ from Def. $17 \neg$ rtsolve $\left(C_{c}, D\right)$

false $\left(C_{s}\right)$

$\Leftrightarrow$ from Def. $11 \exists D \in \operatorname{derivs}(\mathcal{Q})$ s.t. $\neg$ solve $\left(C_{s}, D\right)$

$\Leftrightarrow$ from Def. $99\left(\operatorname{step}(L, D)=\left(\theta, \sigma, \theta^{\prime}\right) \wedge \theta \Rightarrow_{P} \sigma(\right.$ Pre $) \wedge$ $\theta^{\prime} \nRightarrow_{P} \sigma($ Post $\left.)\right)$

$\Leftrightarrow$ from Def. $15 \exists S{ }_{\mathcal{A}}^{*} S^{\prime} \rightsquigarrow \mathcal{A} S^{\prime \prime}$ where

$$
\begin{aligned}
S= & \langle L:: G|\theta|-\rangle \wedge \exists c \# \operatorname{success}(L, \text { Pre }, \text { Post }) \in \mathcal{A}_{C}^{\#}(L) \\
& \wedge \theta \Rightarrow_{P} \text { Pre } \\
S^{\prime}= & \left\langle\operatorname{check}(c):: G\left|\theta^{\prime}\right| \mathcal{E}^{\prime}\right\rangle \wedge \theta^{\prime} \nRightarrow_{P} \text { Post } \\
S^{\prime \prime}= & \left\langle-|-| \mathcal{E}^{\prime \prime}\right\rangle \wedge \mathcal{E}^{\prime \prime}=\mathcal{E}^{\prime} \cup\{\bar{c}\}
\end{aligned}
$$$$
\Leftrightarrow \text { from Def. } 17 \neg \text { rtsolve }\left(C_{s}, D\right)
$$

Th.2 states that assertion condition $C$ is false iff there is a derivation $D$ in which the run-time valuation of the assertion 
condition of $C$ in $D$ is false (i.e., if at least one instance of the assertion condition $A$ is in the error set for such derivation $D)$. Given a set of false assertion conditions we can easily derive the set of false assertions using Def. 8. In order to prove that any assertion is checked this has to be done for all possible derivations for all possible queries, which is often not possible in practice. This is why analysis based on abstractions is often used in practice for this purpose.

\section{Higher-order Assertions on Higher-order Derivations}

Once we have established basic results for the case of firstorder assertions in the context of higher-order derivations, we extend the notion of assertion itself to the higher-order case. The motivation is that in the higher-order context terms can be bound to predicates and our aim is to also be able to state and check properties of such predicates.

\subsection{Anonymous Assertions}

We start by generalizing the notion of assertion to include anonymous assertions: assertions where the predicate symbol is a variable from VS, which can be instantiated to any suitable predicate symbol from PS to produce nonanonymous assertions. An anonymous assertion is an expression of the from ":- pred L : Pre => Post", where $L$ is of the form $X\left(V_{1}, \ldots, V_{n}\right)$ and Pre and Post are DNF formulas of prop literals.

Example 5. The anonymous assertion:

$$
\text { ":- pred } X(A, B) \text { : list }(A) \Rightarrow \operatorname{list}(B) \text {." }
$$

states that any predicate $p \in P$ that $X$ is constrained to should be of arity 2 , it should be called with its first argument instantiated to a list, and if it succeeds, then its second argument should be also a list on success.

We now introduce predprops, which gather a number of anonymous assertions in order to fully describe variables containing higher-order terms (predicate symbols), similarly to how prop literals describe conditions for variables containing first-order terms.

Definition 18. Given Pre $_{i}$ and Post $_{i}$ conjunctions of prop literals, a predprop $p p(X)$ is an expression of the form:

$$
\begin{aligned}
& \operatorname{pp}(\mathrm{X})\left\{\quad:-\operatorname{pred} X\left(V_{1}, \ldots, V_{m}\right): \text { Pre }_{1} \Rightarrow \text { Post }_{1}\right. \text {. } \\
& \text {... } \\
& \left.\because-\operatorname{pred} X\left(V_{1}, \ldots, V_{m}\right): \text { Pre }_{n}=>\text { Post }_{n} .\right\}
\end{aligned}
$$

Definition 19. The corresponding set of anonymous assertion conditions for the predprop $p p(X)$ is defined as $\mathcal{A}_{C}[p p(X)]=\left\{C_{i}[X] \mid i=0 . . n\right\}$ where:

$C_{i}[X]= \begin{cases}\operatorname{calls}\left(X\left(V_{1}, \ldots, V_{m}\right), \text { Pre }\right) & i=0 \\ \operatorname{success}\left(X\left(V_{1}, \ldots, V_{m}\right), \text { Pre }_{i}, \text { Post }_{i}\right) & i=1 . . n\end{cases}$

The variable $X$ can be instantiated to a particular predicate symbol $q \in P S$ to produce a set of non-anonymous assertion conditions $\mathcal{A}_{C}[p p(p)]$ for $q$.

Example 6. Consider defining a comparator (Cmp) predprop that describes predicates of arity 3 which can be used to compare numerical values:

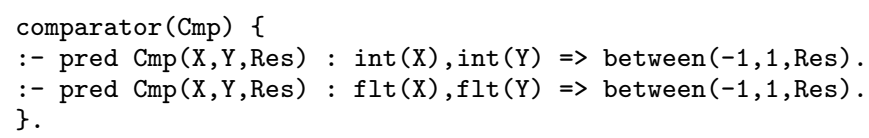

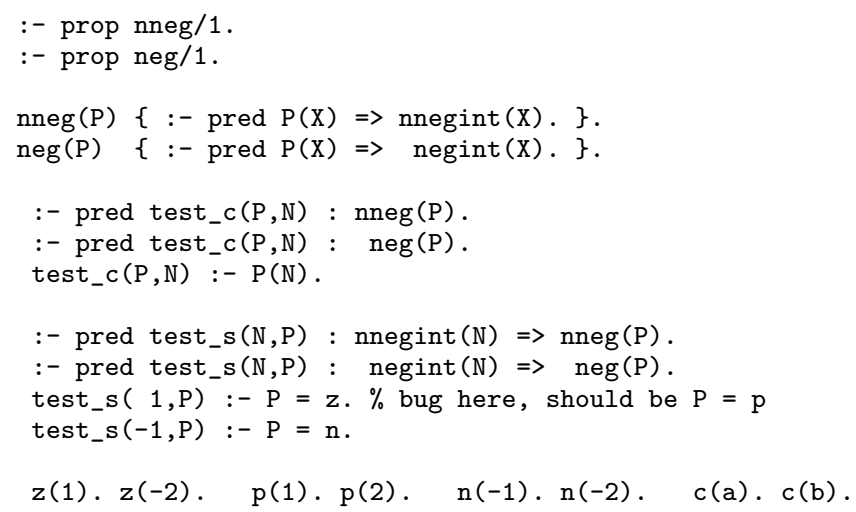

Figure 1. Sample Program with predprops.

The comparator(Cmp) predprop includes two anonymous assertions describing a set of possible preconditions and postconditions for predicates of this kind. In this example:

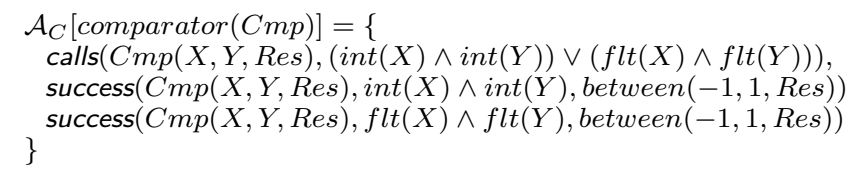

Example 7. Fig. 1 provides a larger example. This example is more stylized for brevity, but it covers a good subset of the relevant cases, used later to illustrate the semantics.

Definition 20. The meaning of a predprop $p p(X)$, denoted $|p p(X)|$ is the set of constraints $\left\{X=q \mid q \in P S, \forall \_\# C \in\right.$ $\mathcal{A}_{C}[p p(q)]$ : checked $\left.(C)\right\}$.

A predicate given by its predicate symbol $p \in \mathrm{PS}$ is compatible with a predprop $p p(X)$ if all the assertions resulting from $p p(p)$ are checked for all possible queries in an annotated program.

\subsection{Operational Semantics for Higher-order Programs with Higher-order Assertions}

We now discuss several alternative operational semantics for higher-order programs with higher-order assertions. In all cases the aim of the semantics is to check whether assertions with predprops hold or not during the computation of the derivations from a query.

\subsubsection{Checking with Static predprops}

According to Definition 20 a predprop literal $p p(X)$ denotes the subset of predicates for which all the associated assertions are checked. When that set of assertions can be statically computed, then $\theta \Rightarrow_{P}$ Cond can be used for both prop and predprop Cond literals, and the operational semantics is identical to the one for the higher-order programs and regular assertions.

We will denote as $S \rightsquigarrow H \mathcal{A}_{\mathbf{s}} S^{\prime}$ a reduction from a state $S$ to a state $S^{\prime}$ under the semantics for higher-order derivations in programs with assertions that may contain higher-order properties, which are statically precomputed. Thus, state reductions are performed as follows:

$$
\frac{\langle G|\theta| \mathcal{E}\rangle \rightsquigarrow \mathcal{A}\left\langle G^{\prime}\left|\theta^{\prime}\right| \mathcal{E}^{\prime}\right\rangle}{\langle G|\theta| \mathcal{E}\rangle \rightsquigarrow H \mathcal{A}_{\mathbf{s}}\left\langle G^{\prime}\left|\theta^{\prime}\right| \mathcal{E}^{\prime}\right\rangle}
$$

The meaning of each predprop, $|p p(X)|$, can be inferred or checked (if given by the user) by static analysis. 
In this semantics, given the program shown in Fig. 1 and the goal test_c $(z,-2)$, assertions are detected to be false since $\{P=\mathbf{z}\} \not \subset|\operatorname{neg}(\mathrm{P})|$ and $\{P=\mathbf{z}\} \not \subset|\operatorname{nneg}(\mathrm{P})|$.

\subsubsection{Checking with Dynamic predprops}

Given the difficulty in determining the meaning of $|p p(X)|$ statically, we also propose a semantics with dynamic checking. We start with an over-approximation of each predprop $|p p(X)|=\{X=p \mid p \in \mathrm{PS}\}$ and incrementally remove predicate symbols, as violations of assertion conditions are detected. :

- we can detect when some assertion condition instance is violated (Def. 15);

- we need a way to obtain a set of assertion condition instances from predprops (anonymous asserion condition instances);

We do that by defining instantiations of anonymous assertion conditions for particular predicate symbols and the dependencies among those instances.

The following two definitions extend the notion of assertion condition instances from Def. 14 to the case of anonymous assertion conditions and higher-order literals:

Definition 21. Given a predprop $p p(X)$ and a predicate symbol $p \in P S, \mathcal{A}_{C}^{\#}[p p(p)]$ denotes the set of labeled hypothetical assertion conditions of the form $h \# C_{p}$, such that $C[X] \in \mathcal{A}_{C}[p p(X)]$ (Def. 19), $L=X\left(V_{1}, \ldots, V_{n}\right), L_{p}=$ $p\left(V_{1}, \ldots, V_{n}\right), C_{p}$ is defined as:

$C_{p}= \begin{cases}\operatorname{calls}\left(L_{p}, \text { Pre }\right) & \text { if } C[X]=\operatorname{calls}(L, \text { Pre }) \\ \operatorname{success}\left(L_{p}, \text { Pre }, \text { Post }\right) & \text { if } C[X]=\operatorname{success}(L, \text { Pre }, \text { Post })\end{cases}$

and $h$ is an identifier that is unique for each $C_{p}$.

In this semantics we allow the assertion condition instances to be derived from the hypothetical assertion conditions in the same way, as in Def. 14. However, the violation of such an instance has to be treated in a special way, as it does not signal the violation of its conditions, but instead of the corresponding predprop. For simplicity, we also introduce a special label $h_{0}$ to denote the assertion conditions that appeared originally in the program. The error set $\mathcal{E}$ in Def. 15 contained negated assertion condition instance identifiers. Now we extend this set with assertion dependency rules of the form $\bigwedge(\bigvee \bar{c}) \rightarrow \bar{c}$. The following definitions provide the description of how such dependencies are generated.

Definition 22. The simplification of a literal $L$ w.r.t. $\theta$ is defined as:

$$
\operatorname{simp}(L, \theta)= \begin{cases}L & \text { if } L \text { is a predprop } \\ \text { true } & \text { if } \theta \Rightarrow_{P} L \\ \text { false } & \text { if } \theta \neq_{P} L\end{cases}
$$

We extend this definition for a conjunction of literals.

Definition 23. Given the label $c$ of an assertion condition instance and a formula of the form Props $=\bigvee_{i=1}^{n}\left(\bigwedge_{j=0}^{m(i)} \operatorname{Prop}_{i j}\right)$, where Prop $_{i j}$ is either a prop or predprop literal, the extension of $\mathcal{A}_{C}$ and $\mathcal{E}$ for dynamic predprop checking, denoted as $\operatorname{ext}\left(\mathcal{A}_{C}, c\right.$, Props $)=\left(\Delta \mathcal{A}_{C}, \Delta \mathcal{E}\right)$, is obtained as follows:

1. if $\operatorname{simp}($ Props, $\theta)=$ false, then $\Delta \mathcal{A}_{C}=\emptyset$ and $\Delta \mathcal{E}=\{\bar{c}\}$
2. otherwise: $\Delta \mathcal{A}_{C}=\bigcup_{i=1}^{n} \mathcal{A}_{C}^{i}$, and

$$
\begin{aligned}
\Delta \mathcal{E}=\{ & \left.\bigwedge_{i=1}^{n}\left(\bigvee_{h \in H_{i}} \bar{h}\right) \rightarrow \bar{c}\right\} \text { where: } \\
\mathcal{A}_{C}^{i}= & \left\{h \# C \in \mathcal{A}_{C}^{\#}\left[\text { Prop }_{i j}\right] \mid 0 \leq j \leq m(i),\right. \\
& \operatorname{Prop}_{i j}=p_{i j}\left(X_{i j}\right), p p_{i j}\left(X_{i j}\right) \text { is a predprop and } \\
& \left.X_{i j} \text { is bound to some } q \in P S\right\} . \\
H_{i}= & \left\{h \mid h \# \#_{-} \in \mathcal{A}_{C}^{i}\right\},
\end{aligned}
$$

We will denote as $S \rightsquigarrow H \mathcal{A}_{\mathrm{d}} S^{\prime}$ a reduction from a state $S$ to a state $S^{\prime}$ under the current semantics.

Definition 24. A state $S=\langle L:: G|\theta| \mathcal{E}\rangle$, where $L$ is a literal can be reduced to a state $S^{\prime}$, denoted $S \rightsquigarrow_{H \mathcal{A}_{d}} S^{\prime}$, as follows:

1. If $L$ is a constraint or $L=X\left(t_{1}, \ldots, t_{n}\right)$, then $S^{\prime}=\left\langle G^{\prime}\right|$ $\theta^{\prime}|\mathcal{E}\rangle$ where $G^{\prime}$ and $\theta^{\prime}$ are obtained in a same manner as in $\langle L:: G \mid \theta\rangle \rightsquigarrow \mathcal{A}\left\langle G^{\prime} \mid \theta^{\prime}\right\rangle$;

2. If $L$ is an atom and $\exists(L:-B) \in \operatorname{defn}(L)$, then for each $c_{i} \# C_{i} \in \mathcal{A}_{C}^{\#}(L)$ :

$$
\begin{aligned}
h_{i} & = \begin{cases}h & \text { if } C_{i} \text { is an instance of some } \\
h \# C \in \mathcal{A}_{C} & \\
h_{0} & \text { otherwise }\end{cases} \\
\left(\Delta_{i} \mathcal{A}_{C}, \Delta_{i} \mathcal{E}\right) & = \begin{cases}\operatorname{ext}\left(\mathcal{A}_{C}, c_{i}, \text { Pre }\right) & \text { if } C_{i}=\operatorname{calls}(L, \text { Pre }) \\
(\emptyset, \emptyset) & \text { otherwise }\end{cases} \\
\text { Post }_{i} & = \begin{cases}\operatorname{check}\left(c_{i}\right) & \text { if } C_{i}=\operatorname{success}\left(L, \text { Pre }_{i}, \text { Post }_{i}\right) \\
\text { true } & \text { and simp }\left(\text { Pre }_{i}, \theta\right)=\text { true }\end{cases}
\end{aligned}
$$

and $S^{\prime}=\left\langle B::\right.$ PostC :: $\left.G|\theta| \mathcal{E}^{\prime}\right\rangle$, where $\mathcal{E}^{\prime}=$ $\mathcal{E} \cup \bigcup_{i}\left\{\bar{c}_{i} \rightarrow \bar{h}_{i}\right\} \cup \bigcup_{i} \Delta_{i} \mathcal{E}, \mathcal{A}_{C}^{\prime}=\mathcal{A}_{C} \cup \bigcup_{i} \Delta_{i} \mathcal{A}_{C}$, and PostC is the sequence Post $C_{1}$ :: ...: $:$ Post $C_{n}$ (simplifying true literals).

3. If $L$ is a check literal check $(c)$ and $c \# \operatorname{success}\left(L^{\prime},{ }_{-}\right.$, Post $) \in$ $\mathcal{A}_{C}^{\#}\left(L^{\prime}\right)$, then $S^{\prime}=\left\langle G|\theta| \mathcal{E}^{\prime}\right\rangle$ where $\left(\Delta \mathcal{E}, \Delta \mathcal{A}_{C}\right)=$ $\operatorname{ext}\left(\mathcal{A}_{C}, c\right.$, Post $), \mathcal{E}^{\prime}=\mathcal{E} \cup \Delta \mathcal{E}$ and $\mathcal{A}_{C}^{\prime}=\mathcal{A}_{C} \cup \Delta \mathcal{A}_{C}$.

Note that in this semantics we support more than one calls assertion condition per predicate (as several predprops may be applied to the same predicate symbol). Also note that in general we cannot prove with dynamic checking that a predprop is true. So, as a safe approximation we treat preconditions in such success assertion conditions as false.

Definition 25. An assertion condition $C$ is trivial if it is of the form calls(_, true) or success(_, _, true). It is also assumed that for any predprop $p p(X) \mathcal{A}_{C}[p p(X)]$ does not contain trivial assertion conditions.

Theorem 3 (Higher-order Run-time Checking). For any annotated program $(P, \mathcal{Q}, \mathcal{A})$, if $\exists D \in \operatorname{derivs}_{H \mathcal{A}}(\mathcal{Q})$ s.t. $\neg$ rtsolve $(C, D) \Rightarrow C \in \mathcal{A}_{C}$ is false.

Proof. In this proof we reflect the case when an assertion condition is falsified because of some of its predprops violation. To do so it is enough to show that at most one predprop was violated. Let us first prove the theorem for the simplified case when the falsified assertion condition is $C_{s}=\operatorname{success}(L$, Pre, $p p(X))$ and then for the case $C_{c}=$ calls $(L, p p(X))$, where $p p(X)$ is a predprop. Without the loss of generality we assume that $\mathcal{A}_{C}[p p(X)]$ has cardinality of 1 (which is a case when $p p(X)$ consists of one anonymous assertion and one of the corresponding anonymous assertion conditions is trivial).

$\neg$ rtsolve $\left(C_{c}, D\right)$

$\Leftrightarrow$ From Def. $17 \exists c^{\prime}, C_{c}^{\prime}, \sigma, L\left(c^{\prime} \# C_{c}^{\prime} \in \mathcal{A}_{C}^{\#}(L)\right) \wedge\left(\sigma\left(C_{c}\right)=\right.$ 


\begin{tabular}{|c|c|c|c|}
\hline G & $\Delta \theta$ & $\Delta \mathcal{E}$ & $\Delta\left(\right.$ labeled instances + hypothetic $\left.\mathcal{A}_{C}\right)$ \\
\hline test_c $(n, X)$ & $\begin{array}{l}P=n \\
N=-1 \\
X=N\end{array}$ & $\begin{array}{l}\bar{c}_{1} \rightarrow \bar{h}_{0} \\
\bar{h}_{1} \wedge \bar{h}_{2} \rightarrow \bar{c}_{1}\end{array}$ & $\begin{array}{l}c_{1} \# \text { calls }(\text { test_c }(n, X), \text { nneg }(n) \vee \text { neg }(n)) \\
h_{1} \# \operatorname{success}(n(Z), \text { true, nnegint }(Z)) \\
h_{2} \# \operatorname{success}(n(Z), \text { true, negint }(Z))\end{array}$ \\
\hline$P(-1)$ & $Z=-1$ & $\begin{array}{l}\bar{c}_{2} \rightarrow \bar{h}_{1} \\
\bar{c}_{3} \rightarrow \bar{h}_{2}\end{array}$ & $\begin{array}{l}c_{2} \# \operatorname{success}(n(-1), \text { true, nnegint }(-1)) \\
c_{3} \# \operatorname{success}(n(-1), \text { true, negint }(-1))\end{array}$ \\
\hline $\begin{array}{l}\operatorname{check}\left(c_{2}\right) \\
\operatorname{check}\left(c_{3}\right)\end{array}$ & - & $\bar{c}_{2}$ & - \\
\hline $\operatorname{check}\left(c_{3}\right)$ & - & - & - \\
\hline$\square$ & - & - & - \\
\hline
\end{tabular}

Table 1. A derivation of the query (test_c $(\mathrm{n}, \mathrm{X})$, true $)$ to the program in Fig. 1

$\left.C_{c}^{\prime}\right) \wedge\left(\mathcal{E}(D) \vdash \bar{c}^{\prime}\right)$

$\Rightarrow$ From Def. 24 and $\mathcal{E}(D) \vdash \bar{c}^{\prime}$ it must hold

$D=\left(\ldots, S_{1}, \ldots, S_{2}, S_{3} \ldots, S_{4}, \ldots\right)$ where:

$$
\begin{aligned}
& S_{1}=\left\langle L^{\prime}::_{-}\left|\theta_{1}\right|{ }_{-}\right\rangle \quad \text { s.t. } \exists L^{\prime}:-B^{\prime} \in \operatorname{defn}(L), \\
& c^{\prime} \# \text { calls }\left(L^{\prime}, \sigma(p p(X))\right) \in \mathcal{A}_{C}^{\#}(L), \\
& \theta_{1} \mid=(X=q), q \in \mathrm{PS} \\
& S_{2}=\left\langle L_{2}::_{-}\left|{ }_{-}\right| \mathcal{E}_{2}\right\rangle \quad \text { s.t. }\left\{\bar{h} \rightarrow \bar{c}^{\prime}, \bar{c}^{\prime} \rightarrow \bar{h}_{0},\right\} \in \mathcal{E}_{2} \text {, } \\
& h \# C_{q} \in \mathcal{A}_{C}^{\#}[p p(q)], L_{2}=q(\ldots) \\
& S_{3}=\left\langle-|-| \mathcal{E}_{3}\right\rangle \\
& S_{4}=\left\langle_{-}|-| \mathcal{E}_{4}\right\rangle \\
& \text { s.t. }\left\{\bar{c}^{\prime \prime} \rightarrow \bar{h}\right\} \in \mathcal{E}_{3}, c^{\prime \prime} \# C_{c}^{\prime \prime} \in \mathcal{A}_{C}^{\#}\left(L_{2}\right) \\
& \text { s.t. } \mathcal{E}_{4} \vdash \bar{c}^{\prime \prime}
\end{aligned}
$$

$\Rightarrow$ From $\mathcal{E}_{3} \vdash \bar{c}^{\prime \prime}$ and Th. 2 we know that $\neg$ checked $\left(C_{c}^{\prime \prime}\right)$ and thus $(X=q) \notin|p p(X)|$ according to Def. 20

$\Rightarrow$ From Def. 6 it follows that $\theta_{3} \nRightarrow_{P} p p(q)$

$\Rightarrow$ Given the state $S_{1}$ before the call to $L^{\prime}$ and the state $S_{3}$ :

$\left(\operatorname{prestep}(L, D)=\left(\theta_{3}, \sigma\right)\right) \wedge\left(\theta^{\prime} \nRightarrow \nexists_{P} \sigma(p p(X))\right)$

$\Rightarrow$ From Def. $9 \neg$ solve $\left(C_{c}, D\right) \Rightarrow$ From Def. 11 false $\left(C_{c}\right)$

$\neg$ rtsolve $\left(C_{s}, D\right)$

$\Leftrightarrow$ From Def. 17. $\exists c^{\prime}, C_{s}^{\prime}, \sigma, L\left(c^{\prime} \# C_{s}^{\prime} \in \mathcal{A}_{C}^{\#}(L)\right) \wedge\left(\sigma\left(C_{s}\right)=\right.$ $\left.C_{s}^{\prime}\right) \wedge\left(\mathcal{E}(D) \vdash \bar{c}^{\prime}\right)$

$\Rightarrow$ From Def. 24 and $\mathcal{E}(D) \vdash \bar{c}^{\prime}$ it must hold

$D=\left(\ldots, S_{1}, S_{2}, \ldots, S_{3}, S_{4}, \ldots, S_{5}, S_{6}, \ldots, S_{7}, \ldots\right)$ where:

$$
\begin{aligned}
& S_{1}=\left\langle L^{\prime}::_{-}\left|\theta_{1}\right|{ }_{-}\right\rangle \quad \text { s.t. } \exists L^{\prime}:-B^{\prime} \in \operatorname{defn}(L) \text {, } \\
& c^{\prime} \# \operatorname{success}\left(L^{\prime}, \sigma(\text { Pre }), \sigma(p p(X))\right) \in \mathcal{A}_{C}^{\#}(L), \\
& \theta_{1} \Rightarrow_{P} \sigma \text { (Pre) } \\
& S_{2}=\left\langle B^{\prime}:: \operatorname{check}\left(c^{\prime}\right)::{ }_{-}\left|{ }_{-}\right| \mathcal{E}_{2}\right\rangle \quad \text { s.t. }\left\{\bar{c}^{\prime} \rightarrow \bar{h}_{0}\right\} \in \mathcal{E}_{2} \\
& S_{3}=\left\langle\operatorname{check}\left(c^{\prime}\right)::_{-}\left|{ }_{-}\right|{ }_{-}\right\rangle \\
& S_{4}=\left\langle-\left|\theta_{4}\right| \mathcal{E}_{4}\right\rangle \\
& S_{5}=\left\langle L_{5}::_{-}\left|{ }_{-}\right|{ }_{-}\right\rangle \\
& S_{6}=\left\langle-|-| \mathcal{E}_{6}\right\rangle \\
& S_{7}=\left\langle{ }_{-}\left|\theta_{7}\right| \mathcal{E}_{7}\right\rangle \\
& \text { s.t. } \theta_{4} \models(X=q), q \in \mathrm{PS} \text {, } \\
& \left\{\bar{h} \rightarrow \bar{c}^{\prime}\right\} \in \mathcal{E}_{4} \\
& h \# C_{q} \in \mathcal{A}_{C}^{\#}[p p(q)] \text {. } \\
& \text { s.t. } L_{5}=q(\ldots) \\
& \text { s.t. }\left\{\bar{c}^{\prime \prime} \rightarrow \bar{h}\right\} \in \mathcal{E}_{6} \text { where } \\
& c^{\prime \prime} \# C_{s}^{\prime \prime} \in \mathcal{A}_{C}^{\#}\left(L_{5}\right) \\
& \text { s.t. } \mathcal{E}_{7} \vdash \bar{c}^{\prime \prime}
\end{aligned}
$$

$\Rightarrow$ From $\mathcal{E}_{7} \vdash \bar{c}^{\prime \prime}$ and Th. 2 we know that $\neg$ checked $\left(C_{s}^{\prime \prime}\right)$ and thus $(X=q) \notin|p p(X)|$ according to Def. 20

$\Rightarrow$ From Def. 6 it follows that $\theta_{7} \nRightarrow_{P} p p(q)$

$\Rightarrow$ Given the state $S_{1}$ before the call to $L^{\prime}$ and the state $S_{7}:\left(\operatorname{step}(L, D)=\left(\theta_{1}, \sigma, \theta_{7}\right)\right) \wedge\left(\theta_{1} \Rightarrow_{P} \sigma(\right.$ Pre $\left.)\right) \wedge\left(\theta_{7} \nRightarrow_{P}\right.$ $\sigma(p p(X)))$ for $c^{\prime} \# C_{s}^{\prime} \in \mathcal{A}_{C}^{\#}(L)$

$\Rightarrow$ From Def. $9 \neg \operatorname{solve}\left(C_{s}, D\right) \Rightarrow$ From Def. 11 false $\left(C_{s}\right)$

Let us trace finished derivations $D^{1}, D^{2}$ and $D^{3}$ from the queries $Q_{1}=($ test_c $(\mathrm{n}, \mathrm{X})$, true $), Q_{2}=($ test_c $(\mathrm{c}, \mathrm{X})$, true $)$ and $Q_{3}=($ (test_s $(1, \mathrm{P}), \mathrm{P}(-2))$, true $)$, respectively, to the program in Fig. 1 .
In $D^{1}{ }_{[1]}$ (see Tab. 1) we encounter two assertions for test_c/2 with a predprop in each precondition and trivial postconditions. According to state reduction rules, $\Delta \mathcal{A}_{C}$ consists of calls assertion condition instance $c_{1}$ and two hypothetical assertion conditions $h_{1}$ and $h_{2}$, derived from predprops nneg/1 and neg/1, and $\Delta \mathcal{E}=\left\{\bar{c}_{1} \rightarrow \bar{h}_{0}, \bar{h}_{1} \wedge \bar{h}_{2} \rightarrow\right.$ $\left.\bar{c}_{1}\right\}$. In $D^{1}{ }_{[2]}$ and current goal $\mathrm{P}(-1)$ (which is implicitly reduced as $\mathrm{n}(-1))$, success assertion condition instances $c_{2}$ and $c_{3}$ are derived from the hypotheses $h_{1}$ and $h_{2}$, and $\Delta \mathcal{E}=\left\{\bar{c}_{2} \rightarrow \bar{h}_{1}, \bar{c}_{3} \rightarrow \bar{h}_{2}\right\}$. Consequently, two check literals, $\operatorname{check}(2)$ and $\operatorname{check}(3)$ are added to the goal sequence. In states $D^{1}{ }_{[3]}$ and $D^{1}{ }_{[4]}$ those literals are reduced, which results in adding $\bar{c}_{2}$ to $\mathcal{E}$ because nnegint $(-1)$ property from the postcondition of $c_{2}$ is violated. This example shows that the mechanism of dependencies between assertion conditions allows to avoid "false negative" results in assertion checking.

The derivation $D^{2}$ is similar to $D^{1}$ (see Tab. 2). The difference is in $D^{2}{ }_{[4]}$ state, when it becomes possible to infer $\mathcal{E} \vdash \bar{c}_{1}$ and thus to conclude that $\mathrm{c} / 1 \notin \mid$ nneg $(X) \mid \wedge \mathrm{c} / 1 \notin$ $|n e g(X)|$ and that both assertions for test_c/2 are false for this query.

In $D^{3}{ }_{[1]}$ (see Tab. 3) we encounter two assertions with a predprop in each postcondition. According to state reduction rules, $\Delta \mathcal{A}_{C}$ for this state consists of calls and success assertion condition instances, $c_{1}$ and $c_{2}, \Delta \mathcal{E}=\left\{\bar{c}_{0} \rightarrow \bar{h}_{0}, \bar{c}_{1} \rightarrow\right.$ $\left.\bar{h}_{0}\right\}$ for them. Also, a check literal $\operatorname{check}\left(c_{1}\right)$ is added to the goal sequence. After its reduction a hypothetical assertion condition $h_{2}$, derived from $\mathrm{nneg}(\mathrm{X})$ predprop, is added to $\mathcal{A}_{C}$ in $D^{3}{ }_{[3]}$, and $\mathcal{E}$ is extended with a dependency rule $\left\{\bar{h}_{2} \rightarrow \bar{c}_{1}\right\}$. In state $D^{3}{ }_{[4]}$ an assertion condition instance $c_{2}$ is obtained from $h_{2}$ and $\Delta \mathcal{E}=\left\{\bar{c}_{2} \rightarrow \bar{h}_{2}\right\}$. Finally, in state $D^{3}{ }_{[5]}$ it becomes possible to infer $\mathcal{E} \vdash \bar{c}_{1}$ and thus detect that the corresponding assertion for test_s $/ 2$ predicate is false because of the predprop nneg $(\mathrm{X})$ violation.

\section{Conclusions and Future Work}

This paper contributes towards filling the gap between higher-order (C)LP programs and assertion-based extensions for error detection and program validation. To this end we have defined a new class of properties, "predicate properties" (predprops in short), and proposed a syntax and semantics for them. These new properties can be used in assertions for higher-order predicates to describe the properties of the higher-order arguments. We have also discussed several operational semantics for performing run-time checking of programs including predprops and provided correctness results.

Our predprop properties specify conditions for predicates that are independent of the usage context. This corresponds 


\begin{tabular}{l|lll}
\hline $\mathrm{G}$ & $\Delta \theta$ & $\Delta \mathcal{E}$ & $\Delta\left(\right.$ labeled instances + hypothetic $\left.\mathcal{A}_{C}\right)$ \\
\hline test_c $(\mathrm{c}, \mathrm{X})$ & $P=c$ & $\bar{c}_{1} \rightarrow \bar{h}_{0}$ & $c_{1} \#$ calls $($ test_c $(c, X)$, nneg $(c) \vee$ neg $(c))$ \\
& $N=a$ & $\bar{h}_{2} \wedge \bar{h}_{3} \rightarrow \bar{c}_{1}$ & $h_{2} \#$ success $(c(Z)$, true, nnegint $(Z))$ \\
& $X=N$ & & $h_{3} \#$ success $(c(Z)$, true, negint $(Z))$ \\
\hline $\mathrm{P}(\mathrm{a})$ & $Z=a$ & $\bar{c}_{2} \rightarrow \bar{h}_{2}$ & $c_{2} \# \operatorname{success}(c(a), \operatorname{true}, \operatorname{nnegint}(a))$ \\
& & $\bar{c}_{3} \rightarrow \bar{h}_{3}$ & $c_{3} \# \operatorname{success}(c(a), \operatorname{true}, \operatorname{negint}(a))$ \\
\hline $\operatorname{check}\left(c_{2}\right)$, & - & $\bar{c}_{2}$ & - \\
check $\left(c_{3}\right)$ & & & \\
\hline $\operatorname{check}\left(c_{3}\right)$ & - & $\bar{c}_{3}$ & - \\
\hline$\square$ & - & - & -
\end{tabular}

Table 2. A derivation of the query (test_c $(c, X)$, true) to the program in Fig. 1

\begin{tabular}{|c|c|c|c|}
\hline $\mathrm{G}$ & $\Delta \theta$ & $\Delta \mathcal{E}$ & $\Delta\left(\right.$ labeled instances + hypothetic $\left.\mathcal{A}_{C}\right)$ \\
\hline $\begin{array}{l}\text { test_s }(1, P), \\
P(-2)\end{array}$ & $N=1$ & $\begin{array}{l}\bar{c}_{0} \rightarrow \bar{h}_{0} \\
\bar{c}_{1} \rightarrow \bar{h}_{0}\end{array}$ & $\begin{array}{l}c_{0} \# \text { calls }(\text { test_s }(1, P), \text { nnegint }(1) \vee \text { negint }(1)) \\
c_{1} \# \operatorname{success}(\text { test_s }(1, P), \text { nnegint }(1), \text { nneg }(P))\end{array}$ \\
\hline $\begin{array}{l}\mathrm{P}=\mathrm{z}, \\
\operatorname{check}\left(c_{1}\right), \\
\mathrm{P}(-2)\end{array}$ & $P=z$ & - & - \\
\hline $\begin{array}{l}\text { check }\left(c_{1}\right), \\
\mathrm{P}(-2)\end{array}$ & - & $\bar{h}_{2} \rightarrow \bar{c}_{1}$ & $h_{2} \# \operatorname{success}(z(Z)$, true, nnegint $(Z))$ \\
\hline$P(-2)$ & $Z=-2$ & $\bar{c}_{2} \rightarrow \bar{h}_{2}$ & $c_{2} \# \operatorname{success}(z(-2)$, true, nnegint $(-2))$ \\
\hline $\operatorname{check}\left(c_{2}\right)$ & - & $\bar{c}_{2}$ & - \\
\hline$\square$ & - & - & - \\
\hline
\end{tabular}

Table 3. A finished derivation of the query ( (test_s $(1, P), P(-2))$, true) to the program in Fig. 1

in functional programming to the notion of tight contract satisfaction [9], and it contrasts with alternative approaches such as loose contract satisfaction [11. In the latter, contracts are attached to higher-order arguments by implicit function wrappers. The scope of checking is local to the function evaluation. Although this is a reasonable and pragmatic solution, we believe that our approach is more general and more amenable for combination with static verification techniques. For example, avoiding wrappers allows us to remove checks (e.g. by static analysis) without altering the program semantics ${ }^{4}$ Moreover, our approach can easily support loose contract satisfaction, since it is straightforward in our framework to optionally include wrappers as special predprops.

We have included the proposed predprop extensions in an experimental branch of the Ciao assertion language implementation. This has the immediate advantage, in addition to the enhanced checking, that it allows us to document higherorder programs in much more accurate way. We have also implemented several prototypes for operational semantics with dynamic predprop checking (see A for a minimalistic implementation), which we plan to integrate into the already existing assertion checking mechanisms for first-order assertions.

\section{References}

[1] H. Aït-Kaci. An Introduction to LIFE - Programming with Logic, Inheritance, Functions and Equations. In D. Miller,

\footnotetext{
$\overline{{ }^{4} \text { E.g. } f(g)=g}$ is not an identity function if wrappers are added to $g$ on call. This complicates reasoning about the program, and may lead to unexpected and hard to detect differences in program semantics. Similar examples can be constructed where the presence of predprops in assertions would invalidate many reasonable program transformations.
}

editor, Proceedings of the 1993 International Symposium on Logic Programming, pages 52-68. MIT Press, 1993.

[2] C. Beierle, R. Kloos, and G. Meyer. A Pragmatic Type Concept for Prolog Supporting Polymorphism, Subtyping, and Meta-Programming. In Proc. of the ICLP'g9 Workshop on Verification of Logic Programs, Las Cruces, Electronic Notes in Theoretical Computer Science, volume 30, issue 1. Elsevier, 2000. URL http://www.elsevier.nl/locate/ entcs/volume $30 . \mathrm{html}$

[3] J. Boye, W. Drabent, and J. Małuszyński. Declarative Diagnosis of Constraint Programs: an assertion-based approach. In Proc. of the 3rd. Int'l Workshop on Automated Debugging-AADEBUG'97, pages 123-141, Linköping, Sweden, May 1997. U. of Linköping Press.

[4] F. Bueno, P. Deransart, W. Drabent, G. Ferrand, M. Hermenegildo, J. Maluszynski, and G. Puebla. On the Role of Semantic Approximations in Validation and Diagnosis of Constraint Logic Programs. In Proc. of the 3rd. Int'l WS on Automated Debugging-AADEBUG, pages 155-170. U. Linköping Press, May 1997.

[5] D. Cabeza. An Extensible, Global Analysis Friendly Logic Programming System. PhD thesis, Universidad Politécnica de Madrid (UPM), Facultad Informatica UPM, 28660Boadilla del Monte, Madrid-Spain, August 2004.

[6] D. Cabeza, M. Hermenegildo, and J. Lipton. Hiord: A Type-Free Higher-Order Logic Programming Language with Predicate Abstraction. In Ninth Asian Computing Science Conference (ASIAN'04), number 3321 in LNCS, pages 93108. Springer-Verlag, December 2004. ISBN ISBN 3-54024087-X.

[7] R. Cartwright and M. Fagan. Soft Typing. In PLDI'91, pages 278-292. SIGPLAN, ACM, 1991.

[8] W. Chen, M. Kifer, and D. Warren. HiLog: A foundation for higher order logic programming. Journal of Logic Programming, 15(3):187-230, 1993. 
[9] C. Dimoulas and M. Felleisen. On contract satisfaction in a higher-order world. ACM Trans. Program. Lang. Syst., 33 (5):16, 2011.

[10] W. Drabent, S. Nadjm-Tehrani, and J. Małuszyński. The Use of Assertions in Algorithmic Debugging. In Proceedings of the Intl. Conf. on Fifth Generation Computer Systems, pages 573-581, 1988.

[11] R. B. Findler and M. Felleisen. Contracts for higher-order functions. In M. Wand and S. L. P. Jones, editors, ICFP, pages 48-59. ACM, 2002. ISBN 1-58113-487-8.

[12] M. Hermenegildo, G. Puebla, and F. Bueno. Using Global Analysis, Partial Specifications, and an Extensible Assertion Language for Program Validation and Debugging. In K. R. Apt, V. Marek, M. Truszczynski, and D. S. Warren, editors, The Logic Programming Paradigm: a 25-Year Perspective, pages 161-192. Springer-Verlag, July 1999.

[13] M. Hermenegildo, G. Puebla, F. Bueno, and P. L. García. Integrated Program Debugging, Verification, and Optimization Using Abstract Interpretation (and The Ciao System Preprocessor). Science of Computer Programming, 58(1-2), 2005.

[14] M. V. Hermenegildo, F. Bueno, M. Carro, P. López, E. Mera, J. Morales, and G. Puebla. An Overview of Ciao and its Design Philosophy. Theory and Practice of Logic Programming, 12(1-2):219-252, January 2012. . http://arxiv.org/abs/1102.5497.

[15] P. Hill and J. Lloyd. The Goedel Programming Language. MIT Press, Cambridge MA, 1994.

[16] C. Laï. Assertions with Constraints for CLP Debugging. In P. Deransart, M. V. Hermenegildo, and J. Maluszynski, editors, Analysis and Visualization Tools for Constraint Programming, volume 1870 of Lecture Notes in Computer Science, pages 109-120. Springer, 2000. ISBN 3-540-41137-2.

[17] L. Lamport and L. C. Paulson. Should your specification language be typed? ACM Transactions on Programming Languages and Systems, 21(3):502-526, May 1999.

[18] G. T. Leavens, K. R. M. Leino, and P. Müller. Specification and verification challenges for sequential object-oriented programs. Formal Asp. Comput., 19(2):159-189, 2007.

[19] F. Logozzo et al. Clousot. http://msdn.microsoft.com/ en-us/devlabs/dd491992.aspx.

[20] E. Mera and J. Wielemaker. Porting and refactoring Prolog programs: the PROSYN case study. TPLP, 13(4-5-OnlineSupplement), 2013.
[21] E. Mera, P. López-García, and M. Hermenegildo. Integrating Software Testing and Run-Time Checking in an Assertion Verification Framework. In 25th International Conference on Logic Programming (ICLP'09), number 5649 in LNCS, pages 281-295. Springer-Verlag, July 2009.

[22] G. Nadathur and D. Miller. Higher-Order Logic Programming. In D. Gabbay, C. Hogger, and A. Robinson, editors, Handbook of Logic in Artificial Intelligence and Logic Programming, volume 5. Oxford University Press, 1998.

[23] L. Naish. Higher-order Logic Programming. Technical Report 96/2, Department of Computer Science, University of Melbourne, Melbourne, Australia, feb 1996. URL: http://www.cs.mu.oz.au/ lee/papers/ho/.

[24] G. Puebla, F. Bueno, and M. Hermenegildo. An Assertion Language for Debugging of Constraint Logic Programs. In Proceedings of the ILPS'97 Workshop on Tools and Environments for (Constraint) Logic Programming, October 1997. Available from ftp://clip.dia.fi.upm.es/pub/papers/ assert_lang_tr_discipldeliv.ps.gz as technical report CLIP2/97.1.

[25] G. Puebla, F. Bueno, and M. Hermenegildo. An Assertion Language for Constraint Logic Programs. In Analysis and Visualization Tools for Constraint Programming, number 1870 in LNCS, pages 23-61. Springer-Verlag, 2000.

[26] G. Puebla, F. Bueno, and M. Hermenegildo. Combined Static and Dynamic Assertion-Based Debugging of Constraint Logic Programs. In Logic-based Program Synthesis and Transformation (LOPSTR'99), number 1817 in LNCS, pages 273-292. Springer-Verlag, March 2000.

[27] Z. Somogyi, F. Henderson, and T. Conway. The Execution Algorithm of Mercury: an Efficient Purely Declarative Logic Programming Language. JLP, 29(1-3):17-64, October 1996.

[28] T. Swift and D. S. Warren. XSB: Extending Prolog with Tabled Logic Programming. TPLP, 12(1-2):157-187, 2012.

[29] S. Tobin-Hochstadt and M. Felleisen. The Design and Implementation of Typed Scheme. In POPL, pages 395-406. ACM, 2008.

[30] D. Warren. Higher-order extensions to Prolog: are they needed? In J. Hayes, D. Michie, and Y.-H. Pao, editors, Machine Intelligence 10, pages 441-454. Ellis Horwood Ltd., Chicester, England, 1982. 


\section{A. Minimalistic Sample Implementation}

The following code (portable to most Prolog systems with minor changes) shows a minimalistic sample implementation (as an interpreter intr/1) of the operational semantics for dynamic predprop checking (Def. 24). Conciseness and simplicity has been favoured over efficiency. We assume that clauses, assertion conditions, and predprops have been parsed and stored in $\mathrm{cl} / 2, \mathrm{ac} / 1, \mathrm{pp} / 2$ facts, respectively. The interpreter will throw an exception the first time that a failed program assertion is detected (see ext $/ 2$ predicate). E.g., $\operatorname{intr}(($ test_s $(1, P), P(1)))$ is a valid query while intr( (test_s $(1, P), P(-2)))$ throws a failed assertion exception. Predicate reset/0 must be called between intr/1 queries to reset error status and temporary data. In the handler errors can be gathered (as in the semantics) or execution aborted.

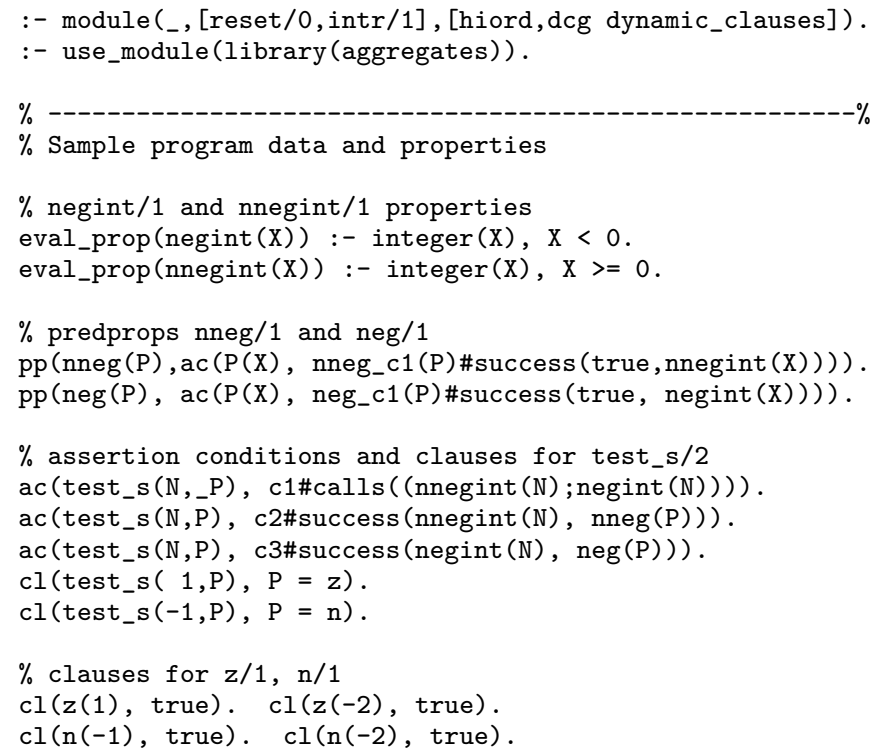

$\%$ predprops nneg/1 and neg/1 $\mathrm{pp}\left(\right.$ nneg $(P)$, ac $\left(P(X)\right.$, nneg_c1 $_{-}(\mathrm{P})$ \#success (true, nnegint $\left.\left.\left.(X)\right)\right)\right)$. $\operatorname{pp}\left(\operatorname{neg}(P), \operatorname{ac}\left(P(X), n_{-} g_{-} 1(P) \#\right.\right.$ success (true, negint $\left.\left.\left.(X)\right)\right)\right)$.

$\%$ assertion conditions and clauses for test_s $/ 2$ ac (test_s (N,_P), c1\#calls ((nnegint $(\mathrm{N})$; negint $(\mathrm{N}))))$. ac (test_s $(N, P), c 2 \# \operatorname{success}(n n e g i n t(N), \operatorname{nneg}(P)))$. ac (test_s $(N, P), c 3 \#$ success $(\operatorname{negint}(N), \operatorname{neg}(P)))$.

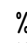

$\%$ Interpreter with higher-order assertion checking $\operatorname{intr}(X):-\operatorname{ctog}(X, X 1)$, !, $\operatorname{intr}(X 1)$.

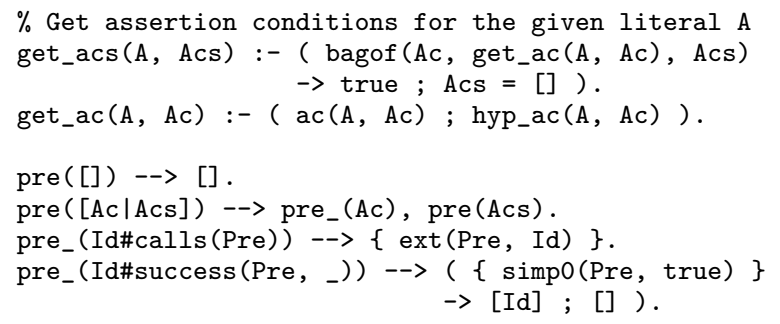

